\title{
Epidemiologic Aspects of Atrial Fibrillation in a Tertiary Hospital in a Sub-Saharan Africa Setting
}

\author{
Félicité Kamdem,2, Ba Hamadou 3,4, Marius Kamdem², Christ Nadège Nganou,4, \\ Anastase Dzudie $^{2,3}$, Yves Monkam², Samuel Kingue ${ }^{3,5}$ \\ ${ }^{1}$ Department of Clinical Sciences, Faculty of Medicine and Pharmaceutical Sciences, University of Douala, Douala, Cameroon \\ ${ }^{2}$ Internal Medicine Service, Cardiology Unit of the Douala General Hospital, Douala, Cameroon \\ ${ }^{3}$ Department of Medicine and Specialties, Faculty of Medicine and Biomedical Sciences, University of Yaoundé I, Yaoundé, \\ Cameroon \\ ${ }^{4}$ Cardiology Unit, Internal Medicine Service, Central Hospital of Yaoundé, Yaoundé, Cameroon \\ ${ }^{5}$ Internal Medicine Service, Cardiology Unit of the Yaoundé General Hospital, Yaoundé, Cameroon \\ Email: drhamadouba@yahoo.fr
}

How to cite this paper: Kamdem, F., Hamadou, B., Kamdem, M., Nganou, C.N., Dzudie, A., Monkam, Y. and Kingue, S. (2017) Epidemiologic Aspects of Atrial Fibrillation in a Tertiary Hospital in a Sub-Saharan Africa Setting. Open Access Library Journal, 4: e3384.

https://doi.org/10.4236/oalib.1103384

Received: January 18, 2017

Accepted: February 19, 2017

Published: February 22, 2017

Copyright $\odot 2017$ by authors and Open Access Library Inc.

This work is licensed under the Creative Commons Attribution International License (CC BY 4.0).

http://creativecommons.org/licenses/by/4.0/

\begin{abstract}
Background: Atrial fibrillation (AF) is the most frequent sustained arrhythmia. The prevalence is increasing with that of other cardiovascular risk factors in our low-income setting. It is potentially serious due to the associated complications. Few data exist on the epidemiology of AF in Sub-Saharan Africa (SSA). Methodology: We carried out a cross-sectional study between 2005 and 2009 in the Cardiology Unit of the Douala General Hospital. Patients were adults $\geq 18$ years of both sex, who had an ECG diagnosis of Atrial Fibrillation and a comprehensive investigation of the cause of AF. Results: A total of 2581 patients were seen in this unit, of whom 182 (7.1\%, [95\%: 6.1 - 8.1]) had AF. Their mean age was $59.2 \pm 15.4$ years, and there were 100 (58.2\%, [95\% CI: 51 - 65.6], $p=0.002$ ) females. Hypertension was the most frequent risk factor (77.3\%, [95\% CI: $70.3-82.8$ ]), and 163 (95.3\%, [95\% CI: 90.7 - 97.8]) of the patients were symptomatic. Hypertensive heart disease (45.6\%, [95\% CI: 38.3 53.1]), idiopathic dilated cardiomyopathy (19.3\%, [95\% CI: $14.1-25.9])$, and valvular heart disease (16.4\%, [95\% CI: 11.6 - 22.6]) were the main etiologic factors of AF. The most frequent complications were heart failure seen in 77 (45\%, [37.8 - 52.5]) and ischemic stroke in 27 (15.5\%, [95\% CI: $11.1-22])$ of patients. Digoxine was prescribed in $121(70.8 \%$, [95\% CI: $63.6-77.1])$ patients to control heart rate, while amiodarone was prescribed in 67 (29.2\%, [95\% CI: 32.2 - 46.7]) patients to revert to sinus rhythm. No electrical cardioversion was carried out. Aspirine was prescribed in 119 (69.6\%, [95\% CI: 62.3 - 76]) patients, and anti-vitamin $\mathrm{K}$ in 53 (30.4\%, [95\% CI: 24.5 - 38.3])
\end{abstract}


patients. Conclusion: Atrial fibrillation was seen in seven percent of patients in the Cardiology Unit of this low-income setting. Patients were relatively young compared with high income settings. Hypertension, idiopathic dilated cardiomyopathy, and valvular heart disease were the main causes. Rate control was the main treatment strategy. Oral anti-coagulants were less frequently used.

\section{Subject Areas}

Cardiology

\section{Keywords}

Epidemiology, Atrial Fibrillation, Cameroon, Sub-Saharan Africa

\section{Introduction}

Cardiovascular disease (CVD) is on the rise in the African continent, accounting for $20 \%$ of cases of death. According to the World Health Organization (WHO), CVD related death will increase by $160 \%$ in 2030 . This will be the first cause of mortality, accounting for 23.6 million deaths [1]. This is due to the rapid urbanization that is associated with changes in life-style, which leads to the emergence of CVD risk such as hypertension, diabetes, dyslipidemia, obesity, tobacco use, and sedentarity [1] [2]. Atrial fibrillation (AF) is the most frequent sustained arrhythmia affecting $3 \%$ of the adult population [3] [4]. It carries a high morbidity and mortality due to its complications such as ischemic stroke, heart failure, and sudden death [5] [6] [7]. The prevalence of AF is on the rise in highincome settings due to the high rates of risk factors such as hypertension, dyslipidemia, heart failure, ischemic heart disease, and valvular heart disease [8]. Due to the rising rate of risk factors of $\mathrm{AF}$ in our setting coupled with the lack of data, this work aimed at studying burden, associated factors, and management of AF in our setting.

\section{Methodology}

Study design and Setting: We carried out this cross-sectional study in the Cardiology unit of the Douala General Hospital (DGH) between January 2006 and December 2009 (47 months). The DGH is a tertiary health institution in Cameroon, sub-Saharan Africa. It has a catchment population of about three million inhabitants. It has vocation providing health care and as a Teaching Hospital for medical students and in-training specialists.

Study population: Participants were adults $\geq 18$ years, of both sex, who were seen in this unit with an electrocardiogram (ECG) documented AF, and who underwent a comprehensive investigation of the cause of AF.

Outcome measures: The main outcome was the presence of Atrial Fibrillation (AF) in the Hospital setting. Secondary outcome measures were the most probable cause of AF after a comprehensive clinical and echocardiographic examination.

Measurements: Complete case records of patients were retrieved from which 
we extracted socio-demographic data (age, sex, and profession), cardiovascular risk factors (hypertension, diabetes, obesity, dyslipidemia, tobacco, and excessive alcohol use), and clinical profile of patients (anthropometry, hemodynamics, symptoms and signs), type and cause of AF, associated heart disease, treatment given, and outcome.

Working definitions: Atrial Fibrillation (AF) was diagnosed based on a documented ECG. The causes of AF were based on the Cardiologists' working diagnosis after a comprehensive clinical evaluation and complementary tests. Paroxystic AF was defined as that which resolved spontaneously within seven days. Persistent AF was defined as that which lasted more than seven days without spontaneous resolution. Permanent AF was defined as that which last for more than one month despite treatment [9].

Sample size: This was a descriptive study of all eligible cases of AF seen during the study period. Thus, the sample size was a convenient sample of all eligible cases of AF.

Statistical analysis: The data was analyzed using SPSS Version 15. We present continuos variables as means \pm standard deviation (SD), and discrete variables as frequencies and proportions, with their 95\% confidence intervals. The difference between means was assessed with the one-way ANOVA, and that between proportions with the Chi-square test or Fischer exact test where appropriate. A $p$ value $<0.05$ was considered statistically significant for observed differences.

Ethical considerations: This work was approved by the ethical committee of the Douala General Hospital. We carried out this work in accordance with the declarations of Helsinki.

\section{Results}

Characteristics of the study population: The flow chart of the study population is shown in Figure 1.

A total of 2581 patients were seen during this period, of whom $182(7.1 \%$, [95\% CI: 6.1 - 8.1]) had AF ( $\approx 3.9$ cases per month or $\approx 46.8$ cases per year). Eleven files were excluded from the final analysis due to insufficient data for a meaningful analysis. Of the 171 complete files, 71 (41.5\%, [95\% CI: 34.4 - 49]) were males and 100 (58.5\%, [95\% CI: 51 - 65.6]) were females $(p=0.002)$. Their mean

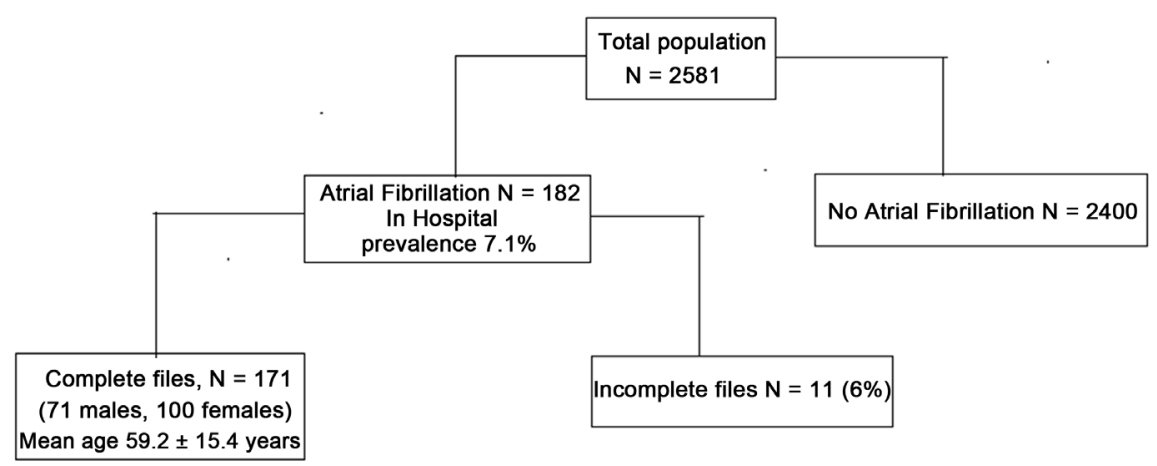

Figure 1. Flow chart of the study population. 
age was $59.2 \pm 15.4$ years, and ranged from 20 to 84 years. Most patients $(26.9 \%$, [95\% CI: 20.8 - 34]) were in the 61 to 70 year old age group (Figure 2). There was no significant difference in the age distribution according to sex (all $p>$ $0.05)$.

The most frequent risk factor was hypertension (77.2\%, [95\% CI: $70.3-82.8])$, and 163 (95.3\%, [95\% CI: 90.7 - 97.8]) of the patients were symptomatic. Table 1 shows patients distribution according cardiovascular risk factors.

The most frequent symptoms were dyspnea (39.9\%, [95\% CI: 32.7 - 47.3]), palpitations (32\%, [95\% CI: 25.6 - 39.5]), and chest pain (14.9\%, [95\% CI: 10.1 20.7]). The distribution of patients according to their symptoms is shown in Table 2.

Permanent AF was seen in 109 (63.7\%, [95\% CI: 56.3 - 70.6]) of patients. The most frequent complications were heart failure seen in 77 (45\%, [37.8 52.5]) of patients, and ischemic stroke in 27 (15.5\%, [95\% CI: 11.1 - 22]). Table 3 shows the distribution of patients according type of atrial fibrillation and its complications.

Table 1. Distribution of patients according cardiovascular risk factors.

\begin{tabular}{ccc}
\hline & Frequency $(\mathrm{n})$ & Percentage $(\%), 95 \%$ CI \\
\hline Hypertension & 132 & $77.2(70.3-82.8)$ \\
Obesity & 21 & $12.3(8.2-18)$ \\
Diabetes & 19 & $11.1(7.2-16.7)$ \\
Alcohol misuse & 15 & $8.8(5.4-14)$ \\
Tobacco & 10 & $5.9(3.2-10.4)$ \\
Dyslipidemia & 9 & $5.3(2.8-9.70)$ \\
\hline
\end{tabular}

Table 2. Distribution of patients according to their symptoms.

\begin{tabular}{ccc}
\hline & Frequency $(\mathrm{n})$ & Percentage (\%), 95\% CI \\
\hline Lipothymia & 11 & $6.4(3.6-11.2)$ \\
Syncope & 12 & $7(4.1-11.90)$ \\
Chest pain & 25 & $14.6(10.1-20.7)$ \\
Palpitation & 55 & $32.2(25.6-39.5)$ \\
Dyspnea & 68 & $39.8(32.7-47.3)$ \\
\hline
\end{tabular}

Table 3. Distribution of patients according type of atrial fibrillation and its complications.

\begin{tabular}{ccc}
\hline & Frequency $(\mathrm{n})$ & Percentage (\%), 95\% CI \\
\hline Type of Atrial Fibrillation & 3 & $1.8(0.6-5)$ \\
Paroxystic & 59 & $34.5(27.8-41.9)$ \\
Persistent & 109 & $63.7(56.3-70.6)$ \\
Permanent & & \\
Complications of Atrial Fibrillation & 77 & $45(37.8-52.5)$ \\
Heart failure & 27 & $15.8(11.1-22)$ \\
Stroke & 1 & $0.6(0.1-3)$ \\
Lower limb ischemia & 6 & $3.5(1.6-7.4)$ \\
Death & &
\end{tabular}


Etiologies of AF: The probable cause(s) of AF (single or in combination) is summarized in Table 4. Hypertensive heart disease (45.6\%, [95\% CI: 38.3 53.1]), idiopathic dilated cardiomyopathy (19.3\%, [95\% CI: $14.1-25.9]$ ), and valvular heart disease (16.4\%, [95\% CI: 11.6 - 22.6]) were the main etiologic factors of AF. Of the 28 patients with valvular heart disease, 17 (60.7\%, [95\% CI: 42.4 - 76.4]) was Rheumatic valvular heart disease, while 11 (39.3\%, [95\% CI: 23.6 - 57.6]) was non-rheumatic valvular heart disease. Structural heart disease was seen in 145 (84.8\%, [95\% CI: 78.7 - 89.4]) patients. Idiopathic dilated cardiomyopathy was more frequently seen in men $(p=0.001)$.

Treatment of AF: This is summarized in Table 5. Digoxine was used in 121 (70.8\%, [95\% CI: 63.6 - 77.1]), and Amiodarone in 67 (39.2\%, [95\% CI: 32.2 46.7]) of patients. No patient underwent electrical cardioversion.

35

30

25

20

15

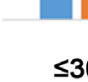

\section{Male Female}
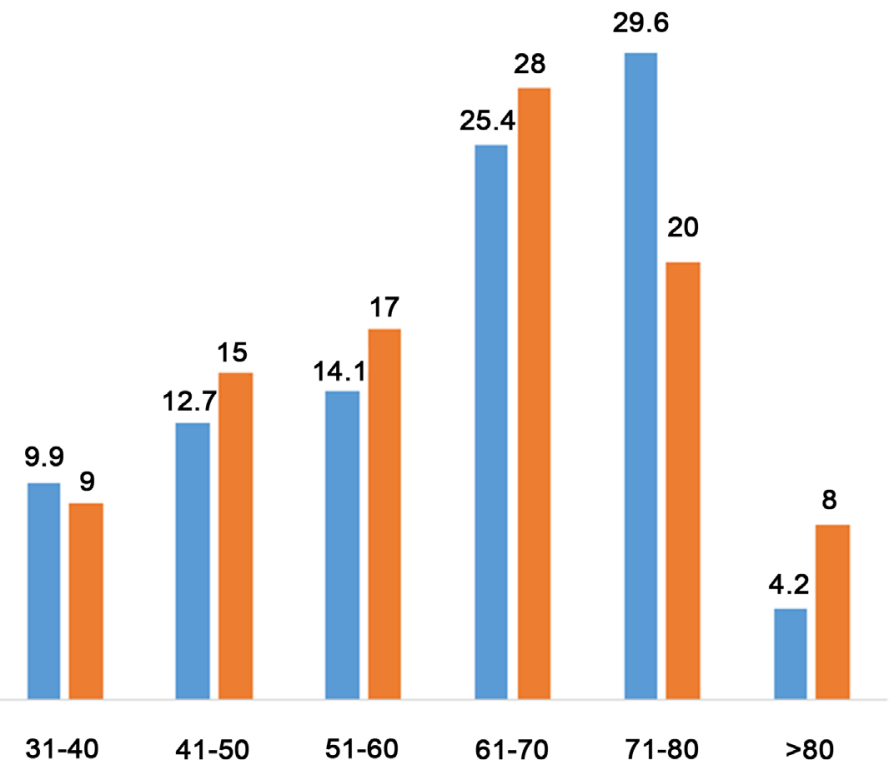

Figure 2. Distribution of patients by age range and sex (all $p>0.05)$.

Table 4. Distribution of patients according the étiologies of AF.

\begin{tabular}{ccccc}
\hline & Overall $(\mathrm{n}=171)$ & Males $(\mathrm{n}=71)$ & Females $(\mathrm{n}=100)$ & $p$ value \\
\hline Hypertensive Heart Disease & $78(45.6)$ & $36(50.7)$ & $42(42)$ & 0.262 \\
Valvular Heart Disease & $28(16.4)$ & $12(16.9)$ & $16(16)$ & 0.876 \\
Dilated Cardiomyopathy & $33(19.3)$ & $22(31)$ & $11(11)$ & 0.001 \\
Hypertrophic Cardiomyopathy & $9(5.3)$ & $5(7)$ & $4(4)$ & 0.387 \\
Ischemic Heart Disease & $8(4.7)$ & $2(2.8)$ & $6(6)$ & 0.330 \\
Pericarditis & $5(2.9)$ & $4(5.6)$ & $1(1)$ & 0.079 \\
Pneumonia & $4(2.3)$ & $2(2.8)$ & $2(2)$ & 0.733 \\
Hyperthyroidism & $2(1.2)$ & $2(2.8)$ & $0(0)$ & 0.093 \\
Alcohol intoxication & $2(1.2)$ & $2(2.8)$ & $0(0)$ & 0.093 \\
Idiopathic & $6(3.5)$ & $4(5.6)$ & $2(2)$ & 0.017 \\
\hline
\end{tabular}


Table 5. Distribution of patients according to the treatment.

\begin{tabular}{ccc}
\hline & Frequency $(\mathrm{n})$ & Percentage $(\%), 95 \% \mathrm{CI}$ \\
\hline Anti-arrhythmia medicine & 121 & $70.8(63.6-77.1)$ \\
Digoxine & 67 & $39.2(32.2-46.7)$ \\
Amiodarone & 7 & $4.1(2-8.2)$ \\
Betabocker & 3 & $1.8(0.6-5)$ \\
Flecainide & & \\
Anti-Thrombotics & 119 & $69.6(62.3-76)$ \\
Aspirin & 53 & $31(24.5-38.3)$ \\
Anti-vitamin K & 47 & $27.5(21.4-34.6)$ \\
Heparin & 57
\end{tabular}

\section{Discussion}

We carried out a cross-sectional study in a sub-Saharan setting to assess the inhospital prevalence, risk factors, and causes of Atrial Fibrillation (AF). The inhospital prevalence of AF was about seven percent, and this was mostly due to hypertensive heart disease. It affected all age groups, especially people in their sixth decades. Heart failure and stroke were the most frequent complications. Most patients were treated with aspirin despite the high thrombo-embolic risk.

Our findings should be interpreted in the light of its limitations. This was a hospital based study in a selected population of patients seen in a cardiology unit. The prevalence reported does not reflect the true population prevalence. The Holter ECG is not used for the diagnosis of AF in our study. The exact burden of AF can only be ascertained with a community based study. The retrospective nature of the study does not permit us to carry out a hormonal investigation of AF for each patient. However, all patients had an echocardiogram to determine the cause of AF (valvular versus non-valvular). Thus, hormonal workup was case-by-case. The possible causes of $\mathrm{AF}$ as reported could represent that of the general population.

The in-hospital prevalence, age, and sex distribution of AF is similar to that reported by other studies in a low-income setting in SSA [10] [11]. The prevalence of AF has been shown to increase with age [12]. More than half of patients with AF were aged greater than sixty years. However, most of the patients had a concomitant structural heart disease that can cause AF. AF appears highly symptomatic, with dyspnea and palpitation being the most frequent symptoms [13]. About half of the patients presented with permanent AF. Twenty five percent of patients with paroxysmal AF on structural heart disease will evolve to persistent or permanent AF [14]. Hypertension was the most frequent risk factor for AF in our setting. This was similar to that reported by the Euro-Heart Survey [15]. Besides hypertension as the main cause of AF in high and low-income settings, the pattern of the aetiology of AF differs slightly with that reported in the Framingham study [6]. Dilated cardiomyopathy and rheumatic valvular heart disease were more frequent causes of AF in our setting. Structural heart disease was similarly 
reported in a low income setting [16]. Conversely, AF has been shown to be a frequent complication of a structural heart disease. Hypertensive heart disease was the most frequent structural heart disease. This corroborates with the high rates of hypertension in the general population [9]. Heart failure and stroke were the most frequent complications of AF. We found relatively fewer cases of heart failure and stroke compared to that reported by Mbolla et al. in a low-income setting [17]. The use of anti-coagulants is a key aspect of treatment of permanent AF. This is to prevent thrombo-embolic events in high risk patients. Most patients were treatedwith aspirin, despite the high risk of thrombo-embolic events. This low use of oral anti-coagulants has been reported by many authors in lowincome settings [12] [13] [16] [17]. This is due to constraints such as the high cost of the new oral anti-coagulants, and the biological surveillance with the anti-vitamin K. Electrical cardioversion was not used in any of the patients. The strategy to control heart rate with digoxin was mostly used in low-income settings as most patients were in heart failure [13] [16] [17]. Betablockers were not frequently used for treating heart failure and controlling heart rate in our setting.

\section{Conclusion}

Atrial fibrillation was seen in seven percent of patients in the Cardiology unit of this low-income setting. Patients were relatively young compared to high income settings. Hypertension, idiopathic dilated cardiomyopathy, and valvular heart disease were the main causes of AF. Rate control was the main treatment strategy used, and oral anti-coagulants was less frequently used despite the high thrombo-embolic risk. Efforts should be heightened to prevent hypertension and rheumatic heart disease in low-income setting. The cost of new oral anti-coagulants or the laboratory tests to monitor the effectiveness of the less expensive anti-vitamin $\mathrm{K}$ should be subsidized.

\section{References}

[1] Bonita, R., Reddy, S., Galbraith, S., et al. (2003) Neglected Global Epidemics: Three Growing Threats: The World Health Report 2003-Shaping the Future. WHO, Geneva, 83-102.

[2] Reddy, K.S. and Yusuf, S. (1998) Emerging Epidemic of Cardiovascular Disease in Developing Countries. Circulation, 97, 596-601.

https://doi.org/10.1161/01.CIR.97.6.596

[3] Bjorck, S., Palaszewski, B., Friberg, L. and Bergfeldt, L. (2013) Atrial Fibrillation, Stroke Risk, and Warfarin Therapy Revisited: A Population-Based Study. Stroke, 44, 3103-3108. https://doi.org/10.1161/STROKEAHA.113.002329

[4] Haim, M., Hoshen, M., Reges, O., Rabi, Y., Balicer, R. and Leibowitz, M. (2015) Prospective National Study of the Prevalence, Incidence, Management and Outcome of a Large Contemporary Cohort of Patients with Incident Non-Valvular Atrial Fibrillation. Journal of the American Heart Association, 4, e001486. https://doi.org/10.1161/jaha.114.001486

[5] Stewart, S., Hart, C.L., Hole, D.J. and McMurray, J.J. (2002) A Population-Based Study of the Longterm Risks Associated with Atrial Fibrillation: 20-Year Follow-Up 
of the Renfrew/Paisley Study. The American Journal of Medicine, 113, 359-364. https://doi.org/10.1016/S0002-9343(02)01236-6

[6] Wolf, P.A., Abbott, R.D. and Kannel, W.B. (1999) Atrial Fibrillation as an Independent Risk Factor for Stroke: The Framingham Study. Stroke, 22, 983-988. https://doi.org/10.1161/01.STR.22.8.983

[7] Krahn, A.D., Manfreda, J., Tate, R.B., Mathewson, F.A. and Cuddy, T.E. (1995) The Natural History of Atrial Fibrillation: Incidence, Risk Factors, and Prognosis in the Manitoba Follow-Up Study. The American Journal of Medicine, 98, 476-484. https://doi.org/10.1016/S0002-9343(99)80348-9

[8] Lousberg, P., Melon, P., Waleffe, A., et al. (2004) Paroxystic Atrial Fibrillation. Revue Médicale de Liege, 59, 301-306.

[9] Lévy, S., Maarek, M. and Coumel, P.H. (1999) On Behalf or the College of French Cardiologists. Characterization of Different Subsets of Atrial Fibrillation in General Practice in France. The ALFA Study. Circulation, 99, 3028-3035. https://doi.org/10.1161/01.CIR.99.23.3028

[10] Mbaye, A., Pessiniba, S., Bodian, M.L., et al. (2010) Frequency, Etiologic Factors, Evolution, and Treatment in a Cardiology Service in Dakar. The Pan African Medical Journal, 6, 16.

[11] Longo, M.B. and Milamba, K.Y. (1993) Epidemiologic and Electrocardiographic Aspects of Atrial Fibrillation in Adults in Congo-Zaïre. L'Information Cardiologique, 17, 227-232.

[12] Lardoux, H., Schaison, F., Jacq, L., et al. (2000) Atrial Fibrillation. AKOS Encyclopédie Pratique de Médecine, EncyclMédChir, 2-0230.

[13] Ntep-Gweth, M., et al. (2010) Atrial Fibrillation in Africa: Clinical Characteristics, Prognosis, and Adherence to Guidelines in CAMEROON. Europace, 12, 482-487. https://doi.org/10.1093/europace/euq006

[14] De Vos, C.B., Pisters, R., Nieuwlaat, R., et al. (2010) Progression from Paroxysmal to Persistent Atrial Fibrillation: Clinical Correlates and Prognosis. Journal of the American College of Cardiology, 55, 725-731. https://doi.org/10.1016/j.jacc.2009.11.040

[15] Nieuwlaat, R., Capucci, A., Camm, A.J., et al. (2005) Atrial Fibrillation Management: A Prospective survey in ESC Member Countries: The Euro Heart Survey on Atrial Fibrillation. European Heart Journal, 26, 2422-2434. https://doi.org/10.1093/eurheartj/ehi505

[16] Coulibaly, I., Anzouan-Kacou, J.B., Konin, C., et al. (2010) Epidemiology and Associated Cardiopathy in the Cardiology Institute of Abidjan. Cardiovascular Journal of Africa, 2, S4-S12.

[17] Mbolla, B.F., Gombet, T., Ikama, M.S., et al. (2006) Atrial Fibrillation: About of 131 Congolese Cases. Medecine d'Afrique Noire, 53, 73-78. 
Submit or recommend next manuscript to OALib Journal and we will provide best service for you:

- Publication frequency: Monthly

- 9 subject areas of science, technology and medicine

- Fair and rigorous peer-review system

- Fast publication process

- Article promotion in various social networking sites (LinkedIn, Facebook, Twitter, etc.)

- Maximum dissemination of your research work

Submit Your Paper Online: Click Here to Submit

Or Contact service@oalib.com 